\title{
Germinación y establecimiento de plantas nativas del matorral tamaulipeco y una especie introducida en un gradiente de elevación
}

\author{
Jaime F. García Pérez ${ }^{1,2}$, Óscar Aguirre Calderón², Eduardo \\ Estrada Castillón ${ }^{2}$, Joel Flores Rivas ${ }^{2,3}$, Javier Jiménez Pérez ${ }^{2}$ \\ y Enrique Jurado Ybarra $^{2}$
}

\section{RESUMEN}

\begin{abstract}
Con el fin de determinar si el calentamiento global podría influir en la ampliación altitudinal de hábitat en el noreste de México, se evaluó la germinación y el crecimiento de cuatro especies de plantas en un gradiente de elevación de 90 a $1960 \mathrm{msnm}$. Los individuos incluyeron tres especies nativas (Cordia boissieri, Ebenopsis ebano y Caesalpinia mexicana) y una introducida, naturalizada en la región (Melia azederach). Se pusieron a germinar en contenedores, semillas de las cuatro especies durante cuatro veces (primavera 2003, 2004; otoño 2003 y 2004), en cinco elevaciones (90, 350, 520, 1,600 y $1,960 \mathrm{msnm}$ ). Las tres elevaciones más bajas incluyen el rango de distribución actual de las especies, y es en éstas en donde en general se presentó la mayor germinación para todas las especies. Para $C$. boissieri fue en las elevaciones de 350, 520 y $1600 \mathrm{msnm}$. Para E. ebano y C. mexicana se obtuvo en 90, 350, 520 y 1600 msnm, y para M. azederach ocurrió a 90, 350, 520 msnm. Además, tanto el crecimiento de las plántulas en altura, como el número de hojas producidas, fueron en general superiores en las elevaciones con mayor porcentaje de germinación. Los resultados sugieren que las especies estudiadas tienen la capacidad para germinar y crecer por encima de su área de distribución actual.
\end{abstract}

PALABRAS CLAVE:

Calentamiento global, elevación, especie exótica, germinación, plantas nativas.

\section{ABSTRACT}

Germination and growth of four plant species were evaluated from 90 to $1960 \mathrm{~m}$ above sea level, with the aim to determine whether global warming could influence growth at higher elevations in northeastern Mexico. Three native species (Cordia boissieri, Ebenopsis ebano and Caesalpinia mexicana) and a naturalized species (Melia azederach) were tested. Seeds were set to germinate in containers four times (Spring 2003, 2004, Autumn 2003 and 2004) at five elevations (90, 350, 520, 1600 and $1960 \mathrm{~m}$ above sea level). The lowest three elevations encompass the current distribution range of the species, and it was in these elevations that in general germination percentage was higher across species. The highest germination for C. boissieri occurred at 350, 520 and $1600 \mathrm{~m}$ above sea level. Highest germination for $E$. ebano and C. mexicana occurred at 90, 350, 520 and $1600 \mathrm{~m}$ above sea level. The highest germination for $M$. azederach occurred at 90,350 and $520 \mathrm{~m}$ above sea level. In addition, seedling growth and number of leaves produced by seedlings were, in general higher at elevations with highest germination percentages. Results presented here suggest that plant species studied have the capacity to germinate and grow at higher elevations from their current distribution.

KEYWORDS:

Global warning, elevation, exotic species, germination, native species.

1 Escuela Preparatoria Número 4 UANL, P. Suárez y V. Carranza, C.P. 67700, Linares, N.L, México ce: fgarcia@fcf.uanl.mx

Facultad de Ciencias Forestales, UANL. A.P. 41, C.P. 67700, Linares, N.L. México

Instituto Potosino de Investigación Científica y Tecnológica, A.C., Camino a la Presa San José No. 2055,

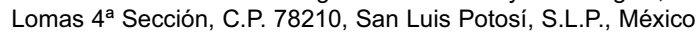




\section{INTRODUCCIÓN}

Los pronósticos de cambio climático sugieren un movimiento de las isotermas del ecuador hacia los polos y hacia lugares de mayor elevación (Hughes, 2000). Esto hace suponer que la distribución actual de las especies podría cambiar altitudinal y latitudinalmente (Grabherr et al., 1994; Parmesan, 1996; Thomas et al., 2001; Walther et al., 2002; Higgins, 2003; Parmesan y Yohe, 2003, Tape et al., 2006). En el mejor de los casos, algunas especies podrían tener la variación genética suficiente como para adaptarse a las modificaciones del ecosistema y así permanecer en sus áreas de distribución actual (Fenner y Thompson, 2005). Sin embargo, existe debate sobre si las especies vegetales podrian dispersarse a la velocidad necesaria para mantener sus poblaciones viables en nuevas altitudes y latitudes (Clark, 1998). Por lo anterior y para fines tanto de conservación como de efectuar plantaciones forestales, sean éstas con fines comerciales o de restauración, es necesario determinar la capacidad adaptativa de las especies para establecerse por arriba de sus límites altitudinales de distribución actual.

En el noreste de México, una amplia extensión del área se encuentra ocupada por matorrales (aproximadamente $200000 \mathrm{~km}^{2}$ ) distribuidos en los estados de Coahuila, Nuevo León, Tamaulipas (Udvary, 1975). El matorral es una vegetación densa de árboles y arbustos con promedio de altura de cuatro metros. Cerca de la mitad de las especies son espinosas (Reid et al., 1990; Jurado et al., 1998; 2000). La mayoría de las especies germinan en otoño y algunas en primavera, coincidiendo con los meses de mayor precipitación pluvial (Flores y Jurado, 1998; Jurado et al., 1998; Jurado et al., 2000; García y Jurado, 2003; Jurado et al., 2006). Los cambios en estos patrones de precipitación pluvial podrían ocasionar modificaciones en la distribución y abundancia de las especies vegetales (Hughes, 2000).

En este estudio se examina el probable desplazamiento en altitud de especies vegetales, en respuesta a los probables efectos de incrementos de temperatura, de acuerdo con los pronósticos del calentamiento global, a lo largo de un gradiente altitudinal, sobre la germinación y crecimiento de plantas de tres especies nativas de matorral (Cordia boissieri "anacahuita", Ebenopsis ebano "ébano" y Caesalpinia mexicana "árbol del potro") y una especie exótica (Melia azederach "canelón"), en tres elevaciones por debajo del límite superior de distribución actual de las especies nativas y dos elevaciones superiores a las de su área de distribución normal.

Las especies nativas seleccionadas en este estudio son abundantes en la región, y su madera ha sido utilizada como postes para cercas y para construcciones rurales (Téllez y Foroughbakhch, 1990; Reid et al., 1990). Se distribuyen desde la Planicie Costera del Golfo hasta elevaciones intermedias de la Sierra Madre Oriental en la que se han observado y registrado las especies. C. mexicana se distribuye en el sur de Texas en EU y en el estado de Nuevo León en el norte de México. Registros en el herbario clave (CFNL) la ubican desde $150 \mathrm{~m}$ hasta $990 \mathrm{~m}$ de altura (Estrada y Marroquín, 1991). C. boissieri también se distribuye en el sur de Texas, y en México se encuentra en los estados de Nuevo León, Tamaulipas, Veracruz, Hidalgo y San Luis Potosí (Vines, 1984); se tienen ejemplares registrados desde 0 hasta $1580 \mathrm{~m}$ de altura en el herbario (CFNL). E. ebano ha sido reportado en Campeche, Yucatán, Nuevo León, Tamaulipas, Veracruz, San Luis Potosí y Sinaloa (Niembro, 1986); los registros en el herbario CFNL ubican a esta especie en 
altitudes entre 40 y $550 \mathrm{msnm}$. Las observaciones de campo del presente estudio, detectaron a las especies desde los 90 $\mathrm{msnm}$, hasta $890 \mathrm{msnm}$ para C. mexicana, $670 \mathrm{msnm}$ para E. ebano y $1530 \mathrm{msnm}$ para C. boissieri.

Estas especies han sido sugeridas por su potencial para programas de rehabilitación de ecosistemas (matorrales) del noreste de México (Alanís, 1981; Heiseke y Foroughbakhch, 1985; Estrada y Marroquín, 1991). La especie exótica es utilizada en la región para fabricación de muebles y se encuentra como especie naturalizada en la zona de estudio a la misma elevación que las especies nativas (Zamarripa, 2002). En el sudeste de Asia, de donde es originaria, se distribuye entre 0 y 2770 msnm (Polunin y Stainton, 1984).

\section{OBJETIVOS}

Los objetivos de este estudio fueron: (i) determinar si la germinación de semi- llas de las cuatro especies ocurre de manera similar en sus rangos de distribución actual y en altitudes superiores a estos; (ii) estimar el crecimiento y la producción de hojas por especie en un gradiente de altitud; y (iii) determinar las elevaciones con potencial para el establecimiento de plantaciones de estas especies. Debido a que los pronósticos de cambio climático preven un eventual desplazamiento altitudinal del clima, en este estudio se plantea que las especies estudiadas tendrán mayor porcentaje de germinación, altura y producción de hojas por encima de sus áreas actuales de distribución

\section{MATERIALES Y MÉTODOS}

\section{Sitios de estudio y especies}

El estudio fue realizado en cinco sitios del noreste de México (Tabla 1); región muy heterogénea en cuanto a su diversidad florística, en la cual se encuen-

Tabla 1. Localidades objeto de estudio en el noreste de México, coordenadas, temperatura promedio anual $\left({ }^{\circ} \mathrm{C}\right)$, precipitación promedio anual $(\mathrm{mm})$ y especies dominantes en los sitios de estudio

\begin{tabular}{|c|c|c|c|c|}
\hline Localidades & Coordenadas & $\begin{array}{c}\text { Temperatura } \\
\left({ }^{\circ} \mathrm{C}\right)\end{array}$ & Precipitación & Especies dominantes \\
\hline Altitud $90 \mathrm{~m}$ & $22^{\circ} 51^{\prime} \mathrm{N}, 98^{\circ} 15^{\prime} \mathrm{W}$ & 23,9 & $912,3-1105$ & $\begin{array}{l}\text { Caesalpinia mexicana A. Gray, Ebenopsis } \\
\text { ebano (Berl.) Barneby \& Grimes, Cordia } \\
\text { boissieri A. DC, Havardia pallens (Benth.) } \\
\text { Brit \& Rose, Randia obcordata Lindley. }\end{array}$ \\
\hline Altitud $350 \mathrm{~m}$ & $24^{\circ} 51^{\prime} \mathrm{N}, 99^{\circ} 35^{\prime} \mathrm{W}$ & 22,3 & $743-1000$ & $\begin{array}{l}\text { Celtis pallida Torr., Cordia boissieri A. DC., } \\
\text { Ebenopsis ebano (Benth.) Coult. , Havardia } \\
\text { pellens (Benth, Brititon \& Rose. }\end{array}$ \\
\hline Altitud $520 \mathrm{~m}$ & $24^{\circ} 37^{\prime} \mathrm{N}, 99^{\circ} 49^{\prime} \mathrm{W}$ & 22,0 & $743-1000$ & $\begin{array}{l}\text { Celtis pallida Torr., Cordia boissieri A. DC., } \\
\text { Ebenopsis ebano (Benth.) Coult., Havardia } \\
\text { palllens (Benth.. Britton \& Rose, Prosopis } \\
\text { laevigata (Humb \& Bonpl. ex. Wild.) M.C. } \\
\text { Johnston }\end{array}$ \\
\hline Altitud $1600 \mathrm{~m}$ & $26^{\circ} 41^{\prime} \mathrm{N}, 99^{\circ} 51^{\prime} \mathrm{W}$ & 17,6 & 550 a 600 & $\begin{array}{l}\text { Pinus cembroides Zucc., P. greggii } \\
\text { Engelm. ex Parl., Juniperus flaccida } \\
\text { Schltdl., J. deppeana Steud., Yucca } \\
\text { carrerosana (Trel.) Mckelvey y Arbutus } \\
\text { xalapensis Kunth } \\
\text { Agave sp., Ceanothus greggii A. Gray y } \\
\text { Salvia regla Cav. }\end{array}$ \\
\hline Altitud $1960 \mathrm{~m}$ & $26^{\circ} 52^{\prime} \mathrm{N}, 100^{\circ} 10^{\prime} \mathrm{W}$ & 15,7 & 550 a 600 & 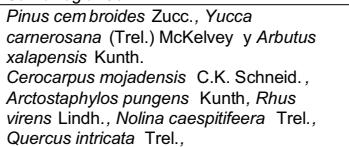 \\
\hline
\end{tabular}

Los datos de temperatura y precipitación fueron proporcionados por la Comisión Nacional del Agua (CNA).

La exposición y pendiente de los sitios donde fueron depositados los contenedores con las semillas, se mantuvieron constantes a lo largo de los cuatro ensayos. 
tran desde matorrales localizados de 100 a $850 \mathrm{msnm}$ hasta bosques de encinos y coníferas distribuidos entre 900 y 3,550 msnm (Estrada y Marroquín, 1991).

Para evaluar la hipótesis planteada y la probable tendencia de las especies estudiadas a germinar y establecerse en mayores altitudes, el estudio fue realizado a lo largo de un gradiente altitudinal. Se pusieron a germinar semillas en cinco sitios con distinta altitud, tomándose la germinación como la emergencia de plántulas del suelo. Las especies utilizadas fueron C. mexicana A. Gray (Caesalpiniaceae), C. boissieri A. DC (Boraginaceae) y E. ebano (Berl.) Barneby \& Grimes (Mimosaceae), tres nativas de matorral espinoso tamaulipeco, así como $M$. azederach $\mathrm{L}$ (Meliaceae), especie introducida que crece naturalmente en el rango altitudinal probado (0-2 $770 \mathrm{msnm}$ en el sureste asiático; Polunin y Stainton, 1984). Tres de los sitios que se seleccionaron se encuentran dentro de la distribución normal de las especies nativas y los dos restantes presentan una altitud mayor.

El ensayo se estableció en las épocas del año que coinciden con la germinación y el establecimiento de especies nativas en la región (Flores y Jurado, 1998; Jurado et al., 1998, 2006; García y Jurado, 2003). Con el fin de incluir la variación ambiental interanual, el ensayo fue realizado durante dos años consecutivos.

\section{Tratamiento de semillas}

Las semillas fueron colectadas durante la primavera y el verano del 2002. La colecta fue realizada en un rodal de al menos 20 plantas madre con el fin de abarcar la variación genética natural de las poblaciones. Se seleccionaron árboles fenotípicamente superiores y se tuvo cuidado de que las semillas fueran de apariencia sana, que no mostraran evidencias de crecimiento de hongos o de parasitismo por insectos. Las semillas fueron mezcladas y puestas a secar en papel estraza a temperatura ambiente por un período de dos meses con el fin de permitir su maduración, antes de que las pruebas de germinación fueran realizadas. No se realizaron pruebas de viabilidad, ni se utilizaron fungicidas. Las semillas fueron escarificadas mecánicamente con papel lija (excepto las semillas de Caesalpinia mexicana), esta técnica es considerada la más eficiente para promover la germinación de semillas de especies de cubierta dura del noreste de México (Foroughbakhch, 1989; Flores y Jurado, 1998).

Después de haber sido escarificadas, las semillas fueron sembradas en contenedores (una semilla por contenedor) de poliestireno de $17 \mathrm{~cm}$ de diámetro $\times 27 \mathrm{~cm}$ de profundidad y cubiertas con $5 \mathrm{~mm}$ de suelo. Se utilizó sustrato a base de tierra y hojarasca proveniente de una zona de matorral tamaulipeco de Linares N.L. La frecuencia de riego fue de dos veces por semana y tres en los días con temperatura mayor de $35^{\circ} \mathrm{C}$, con aproximadamente $200 \mathrm{ml}$ por riego. Los contenedores fueron colocados bajo cobertura de vegetación natural en cada uno de los sitios (Tabla 1).

\section{Diseño experimental}

Se realizaron seis repeticiones de 20 semillas (una semilla por contenedor) en cada uno de los sitios, durante cuatro temporadas. Las variables de respuesta estudiadas fueron: (i) Porcentaje de emergencia de plántulas, expresado como el porcentaje de plántulas emergidas a los 45 días posteriores a la siembra; (ii) Altura de plantas y (iii) Número de hojas. En cada uno de los sitios, la evaluación de la emergencia se 
realizó cada cuatro días hasta el día 45 y después cada quince días, hasta completar la emergencia de plántulas. La longitud de plantas y el número de hojas fueron registrados cada 15 días en cada uno de los sitios, por un período de siete meses.

\section{Análisis estadísticos}

Las variables de respuesta obtenidas (porcentaje de emergencia, altura de plántulas y número de hojas) fueron analizadas mediante ANVA de dos factores, siendo los factores la altitud (cinco niveles) y la temporada (cuatro niveles). Antes de realizar los ANVA, los datos de porcentaje de emergencia fueron normalizados utilizando la transformación del arcoseno de la raíz cuadrada (Sokal y Rohlf, 1994). Para realizar los análisis estadísticos de longitud de plantas y número de hojas se utilizaron los datos obtenidos en la última medición (a los siete meses). Después de los ANVA, se realizaron pruebas de Tukey para determinar diferencias entre tratamientos.

\section{RESULTADOS Y DISCUSIÓN}

\section{Porcentaje de emergencia de plántulas}

De las 9600 semillas sembradas (2 400 por especie), emergieron un total de 4519 a los 45 días (47,07\%), observándose plántulas emergidas durante las cuatro temporadas en las cinco altitudes. También se encontraron evidencias de depredación por herbívoros en hojas y tallos, pero solamente cinco plantas perecieron.

En la Tabla 2 se presentan los resultados de plántulas emergidas por temporada en las cinco altitudes estudiadas. C. mexicana tuvo en total 1436 semillas germinadas, con un porcentaje de emergencia $(\% \mathrm{E})=59,83 \%$. Esta especie mostró el mayor número de plántulas emergidas, mientras que C. boissieri tuvo el valor más bajo (934 plántulas, equivalente al 38,91\%). El número de plántulas emergidas de $E$. ebano fue de 1165 , para un $\% \mathrm{E}$ de $48,54 \%$. Finalmente, emergieron 984 plantas de $M$. azederach, lo que representa un $\% E=41,00 \%$.

El ANVA de dos factores para porcentaje de emergencia de plántulas de cada una de las especies, mostró diferencias estadísticas significativas entre temporadas $(P<0,001 ; G . L .=3)$, entre altitudes $(P<0,001 ; G . L .=4)$ y en la interacción altitud $X$ temporada $(P<0,001 ;$ G.L. $=12)$. La emergencia de plántulas fue mayor en primavera y registró valores menores en otoño en todas las altitudes, con valores más bajos en altitudes mayores a las áreas de distribución natural de las especies, probablemente a causa de bajas temperaturas (Figura 1).

Los mayores porcentajes de emergencia de plántulas de C. mexicana (Tabla 3, Figuras 2 a-d), se obtuvieron en el área de distribución natural de la especie (altitudes de 90, 350 y $520 \mathrm{~m}$ ) y por encima de ésta (1600 m). El más bajo porcentaje de emergencia de plántulas se presentó a 1960 msnm, aún y cuando esta especie ha sido detectada en el altiplano mexicano en elevaciones superiores a 2000 msnm (Estrada y Marroquín, 1991).

Para C. boissieri los porcentajes de emergencia de plántulas más altos se presentaron en altitudes de 520 y 1600 msnm, dentro y por encima de su área de distribución normal (Tabla 3, Figuras 3 ad), seguidos de los obtenidos a 350 y 90 msnm; el porcentaje de emergencia más bajo se registró a una altitud de 1960 msnm.

En el caso de E. ebano, el mayor porcentaje de emergencia de plántulas (Tabla 3, Figuras 4 a-d) se presentó en su 
Tabla 2. Número de plantas emergidas por especie y temporada en las cinco localidades estudiadas

\begin{tabular}{|l|c|c|c|c|}
\hline \multirow{2}{*}{ Especie } & \multicolumn{4}{|c|}{ Número de plantas emergidas } \\
\cline { 2 - 5 } & $\begin{array}{c}\text { Primavera } \\
2003\end{array}$ & $\begin{array}{c}\text { Otoño } \\
2003\end{array}$ & $\begin{array}{c}\text { Primavera } \\
2004\end{array}$ & $\begin{array}{c}\text { Otoño } \\
2004\end{array}$ \\
\hline C. mexicana & 390 & 246 & 527 & 273 \\
\hline C. boissieri & 240 & 193 & 364 & 137 \\
\hline E. ebano & 338 & 256 & 425 & 146 \\
\hline M. azederach & 271 & 172 & 321 & 220 \\
\hline
\end{tabular}
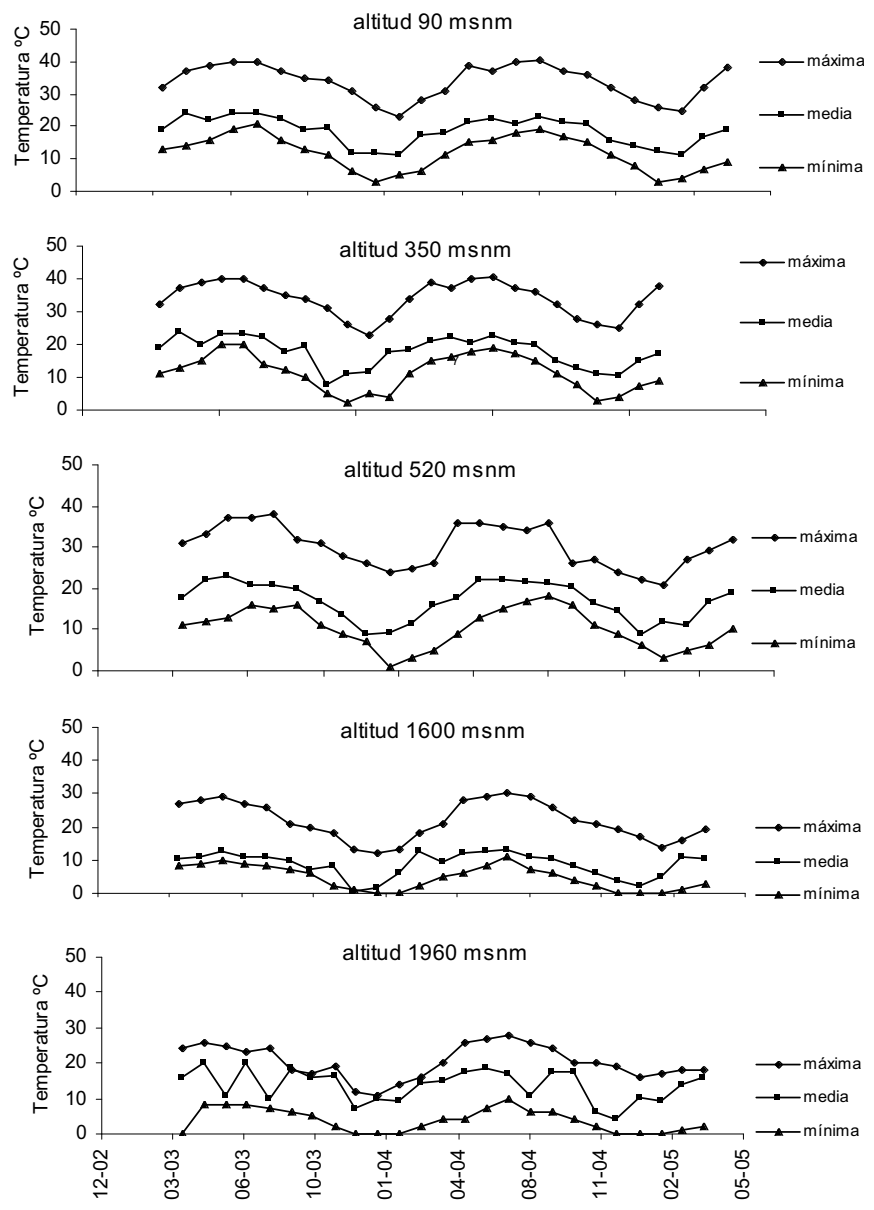

Figura 1.

Temperaturas máximas, medias y mínimas, que se presentaron de abril 2003 a abril 2005, en cinco altitudes (90-1960 m) en el noreste de México.

Los datos fueron proporcionados por la Comisión Nacional del Agua (CNA). 
Tabla 3. Porcentaje de emergencia de plantas a los 45 días de cuatro especies en cinco localidades de distinta altitud.

\begin{tabular}{|c|c|c|c|c|c|c|c|c|}
\hline \multirow{3}{*}{$\begin{array}{l}\text { Altitud } \\
\text { (m) }\end{array}$} & \multicolumn{8}{|c|}{ Porcentaje de emergencia de plantas } \\
\hline & \multicolumn{2}{|c|}{ C. mexicana } & \multicolumn{2}{|c|}{ C. boissieri } & \multicolumn{2}{|l|}{ E. ebano } & \multicolumn{2}{|c|}{ M. azederach } \\
\hline & $\begin{array}{l}\text { Mayor } \\
\text { promedio }\end{array}$ & $\begin{array}{l}\text { Menor } \\
\text { promedio }\end{array}$ & $\begin{array}{l}\text { Mayor } \\
\text { promedio }\end{array}$ & $\begin{array}{l}\text { Menor } \\
\text { promedio }\end{array}$ & $\begin{array}{l}\text { Mayor } \\
\text { promedio }\end{array}$ & $\begin{array}{l}\text { Menor } \\
\text { promedio }\end{array}$ & $\begin{array}{l}\text { Mayor } \\
\text { promedio }\end{array}$ & $\begin{array}{l}\text { Menor } \\
\text { promedio }\end{array}$ \\
\hline 90 & 97,5 a & $50,0 \mathrm{df}$ & $60,0 \mathrm{bc}$ & 41,6 ef & $73,3 \mathrm{abc}$ & $42,5 \mathrm{fg}$ & 64,1 a & $25,5 \mathrm{~cd}$ \\
\hline 350 & 99,1 a & $50,0 \mathrm{df}$ & $66,6 a b$ & $33,3 \mathrm{fg}$ & 82,5 a & $39,1 \mathrm{fg}$ & $62,5 a b$ & 28,9 bc \\
\hline 520 & 98,3 a & $58,8 \mathrm{ce}$ & $71,1 \mathrm{a}$ & $32,5 \mathrm{~g}$ & $77,5 \mathrm{ab}$ & $49,1 \mathrm{de}$ & $64,1 \mathrm{a}$ & 28,7 bc \\
\hline 1600 & 97,5 a & $28,3 \mathrm{hi}$ & 69,1 a & $5,83 \mathrm{hi}$ & $71,6 \mathrm{abc}$ & $14,1 \mathrm{hj}$ & 50,0 de & 3,64 ef \\
\hline 1960 & 47,5 ef & $4,16 \mathrm{i}$ & $35,8 \mathrm{fg}$ & $0,83 \mathrm{i}$ & $49,1 \mathrm{de}$ & $2,50 \mathrm{j}$ & $5,0 \mathrm{~h}$ & $1,56 \mathrm{f}$ \\
\hline
\end{tabular}

área normal de distribución natural, a 350 msnm, seguido del sitio adyacente a 520 $\mathrm{msnm}$. Menores porcentajes fueron registrados a altitudes de 90 y 1600 msnm, el más bajo porcentaje fue registrado a $1960 \mathrm{msnm}$.

La especie introducida $M$. azederach presentó mayor emergencia de plántulas en las localidades a 90 y 520 msnm (Tabla 3, Figuras 5 a-d) y menor en la localidad de mayor altitud.

El suministro de humedad durante el estudio fue constante, por lo que el patrón de emergencia de plántulas fue dependiente de la temperatura. En el área natural de distribución de las especies se presentaron temperaturas altas similares de primavera a otoño (Figura 1), siendo menores de primavera a verano tardío en la altitud de 1 $600 \mathrm{msnm}$. Las más bajas ocurrieron a $1960 \mathrm{msnm}$. Para las cuatro especies, los porcentajes más altos de emergencia de plántulas se presentaron en la temporada primavera 2004, dentro del área de distribución natural de las especies, en las altitudes de 90 a 520 msnm y en la de $1600 \mathrm{msnm}$. El menor porcentaje de emergencia se obtuvo a $1960 \mathrm{msnm}$, sitio asociado a tempera- turas bajas que inhiben la germinación de las especies estudiadas (Figura 1).

La humedad y las altas temperaturas que se presentaron al inicio de primavera y que persistieron hasta otoño tardío en el área de distribución natural de las especies (a 90, 350 y $520 \mathrm{msnm}$ ), y hasta el final del verano en los sitios localizados a 1600 y 1960 msnm, estimularon la germinación de plántulas dentro de su área de distribución natural (a 90, 350 y 520 msnm) y por encima de ésta (a 1600 msnm). En contraste con nuestros resultados de germinación, los estudios de Pérez-García et al. (1995), Escudero et al. (1997), y Pérez-García y GonzálezBenito (2005); encontraron que las bajas temperaturas (alrededor de $15^{\circ} \mathrm{C}$ ), estimularon la germinación de semillas de especies de plantas del Mediterráneo.

Los menores porcentajes de emergencia de plántulas para las cuatro especies se registraron las temporadas de otoño 2003 y 2004 en las altitudes de 1600 y 1960 msnm (Figura 1), debido probablemente a la inhibición de la germinación por bajas temperaturas (Thompson y Grime, 1983; Hilhorst y Karssen, 2000; Flores y Briones, 2001). Así, nuestros resultados apoyan a Holt- 

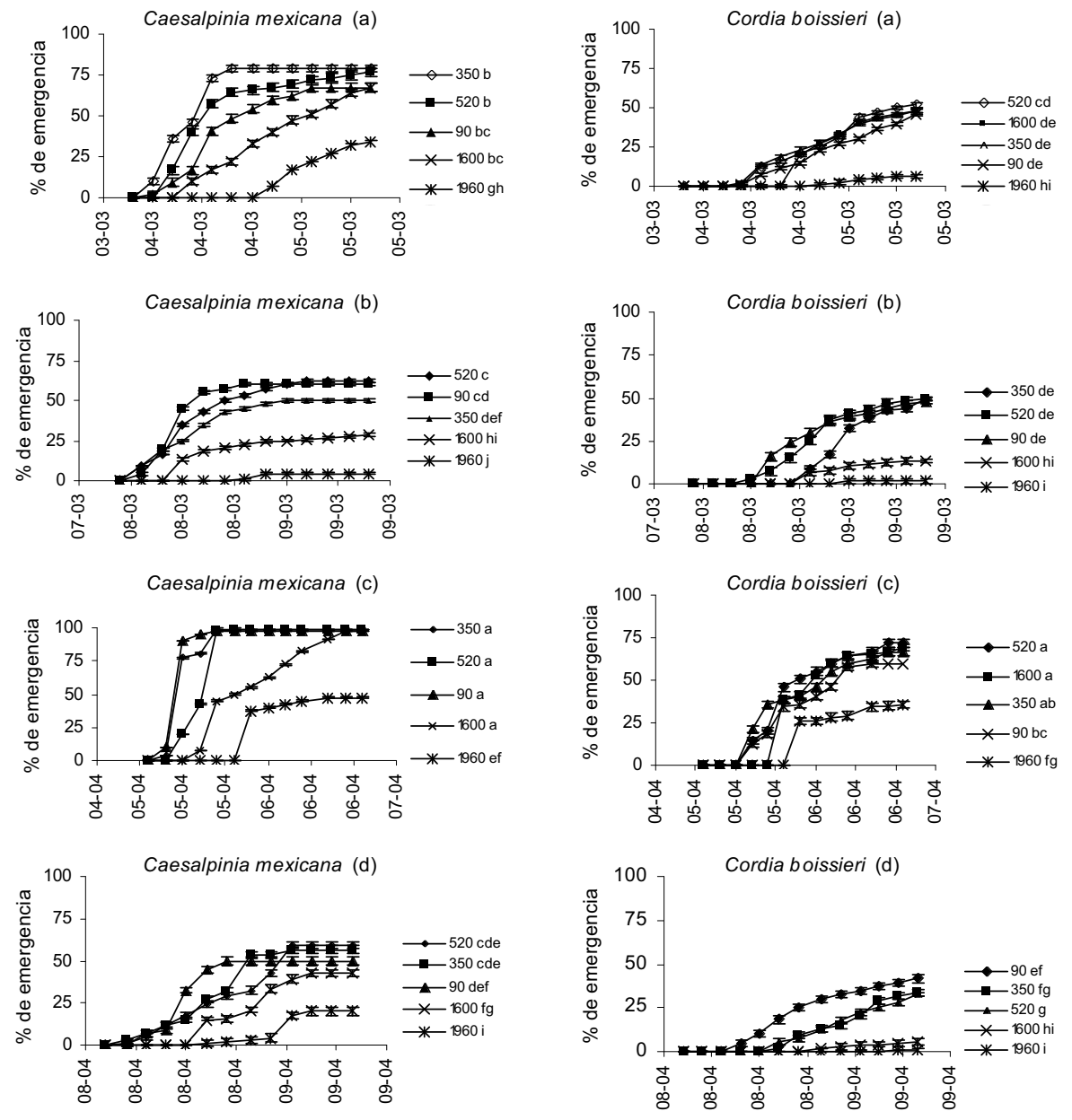

Figura 2.

Porcentaje de emergencia de plantas acumulada, a los 45 días para $\mathrm{C}$. mexicana, por cuatro temporadas (a-d), en cinco altitudes, de 90 a 1960 msnm. Cada punto representa los promedios de porcentaje de germinación acumulada, en seis repeticiones de 20 semillas por cada repetición. Letras diferentes indican diferencia estadística significativa, de acuerdo a pruebas de comparación de medias de Tukey $(\alpha=0,05)$ realizada a

los 45 días. Las barras de error (+/-) representan intervalos de confianza para cada media $(\alpha=0,05)$.

Figura 3.

Porcentaje de emergencia de plantas acumulada, a los 45 días para $C$.

boissieri por cuatro temporadas (a-d), en cinco altitudes, de 90 a 1960 msnm.

Cada punto representa los promedios de porcentaje de germinación acumulada, en seis repeticiones de 20 semillas por cada repetición. Letras diferentes indican diferencia estadística significativa, de acuerdo a pruebas de comparación de medias de Tukey $(\alpha=0,05)$ realizada a

los 45 días. Las barras de error (+/-) representan intervalos de confianza para cada media $(\alpha=0,05)$. 

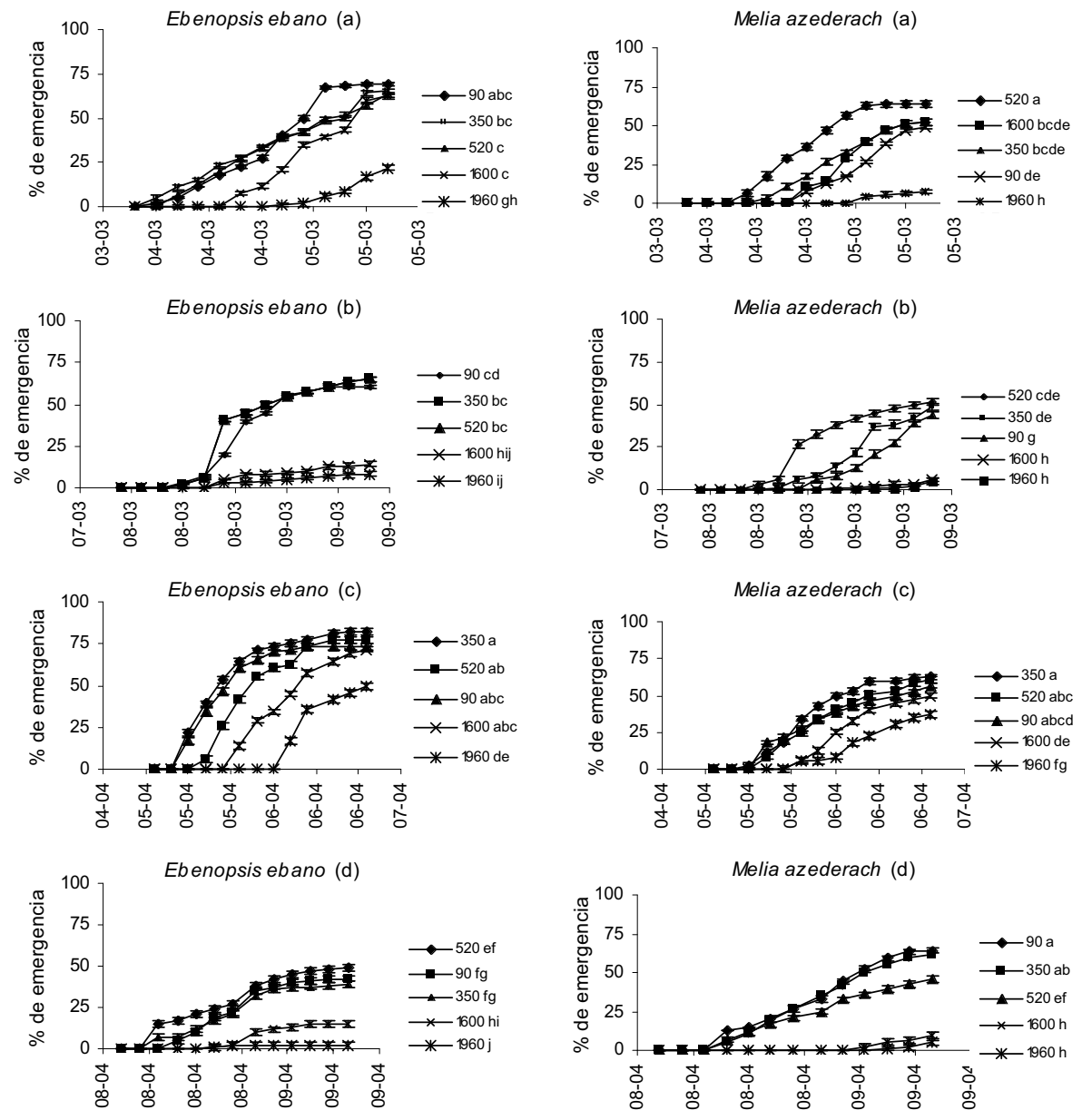

Figura 4.

Porcentaje de emergencia de plantas acumulada, a los 45 días para E. ebano, por cuatro temporadas (a-d), en cinco altitudes, de 90 a 1960 msnm. Cada punto representa los promedios de porcentaje de germinación acumulada, en seis repeticiones de 20 semillas por cada repetición. Letras diferentes indican diferencia estadística significativa, de acuerdo a pruebas de comparación de medias de Tukey $(\alpha=0,05)$ realizada a los 45 días. Las barras de error (+/-) representan intervalos de confianza para cada media $(\alpha=0,05)$.

Figura 5.

Porcentaje de emergencia de plantas acumulada, a los 45 días para $\mathrm{M}$. azederach, por cuatro temporadas (a-d), en cinco altitudes, de 90 a 1960 msnm. Cada punto representa los promedios de porcentaje de germinación acumulada, en seis repeticiones de 20 semillas por cada repetición. Letras diferentes indican diferencia estadística significativa, de acuerdo a pruebas de comparación de medias de Tukey $(\alpha=0,05)$ realizada a los 45 días. Las barras de error (+/-) representan intervalos de confianza para cada media $(\alpha=0,05)$. 
meier y Broll (2005), quienes mencionan al clima como la principal fuerza selectiva en las poblaciones naturales.

\section{Longitud de plantas (mm)}

El ANVA de dos factores para longitud de plantas de cada una de las especies, mostró diferencias estadísticas significativas entre temporadas $(P<0,001 ; G . L .=$ $3)$, siendo mayor el valor obtenido en la primavera 2004. También se encontraron diferencias entre altitudes $(P<0,001$; G.L. $=4)$ y en la interacción altitud por temporada $(P<0,001 ;$ G.L. $=12)$.

El crecimiento de C. mexicana (Tabla 4, Figura 6 a-d) y de E. ebano (Tabla IV, Figura 8 a-d) fue mayor en las cuatro primeras elevaciones $(90,350,520$ y $1600 \mathrm{msnm}$ ) que en la elevación de $1960 \mathrm{msnm}$. En cambio, el crecimiento de $C$. boissieri (Tabla 4, Figura 7 a-d) fue mayor en las elevaciones intermedias (350 y $520 \mathrm{msnm}$ ), con menores promedios de crecimientos a 90 y 1,600 msnm; el menor crecimiento se obtuvo también en la mayor altitud. Por otra parte, $M$. azederach presentó mayor crecimiento en las altitudes de 90,350 y 520 msnm que en la altitud de $1600 \mathrm{msnm}$. En la altitud de 1960 msnm el crecimiento fue aún menor (Tabla 4, Figura 9 a-d).

En general, las plantas mostraron mayor crecimiento en primavera de 2004 y menor crecimiento en altitudes mayores a las áreas de distribución natural de las especies. Las cuatro especies mostraron mayor crecimiento en sus áreas de distribución normal ( 90,350 y $520 \mathrm{msnm})$. A $1600 \mathrm{msnm}$ se registró mayor crecimiento de plantas que a $1960 \mathrm{msnm}$, debido probablemente a que en esta última elevación las temperaturas son menores (Figura 1). Este resultado puede también deberse a diferencias en radiación (Bret-Harte et al., 2004; Castro et al.,
2004). El sitio a $1600 \mathrm{msnm}$ muestra condiciones para crecimiento hasta otoño temprano. Kullman (2002) menciona que las condiciones modificadas producidas por el calentamiento global, como inviernos suaves, favorecen el establecimiento y crecimiento de plantas, con reducido riesgo de desecación por heladas.

\section{Número de hojas}

El ANVA de dos factores para el número de hojas de cada una de las especies mostró diferencias estadísticas significativas entre temporadas $(P<$ $0,001 ;$ G.L. $=3)$, entre altitudes $(P<$ 0,$001 ; G . L .=4$ ) y en la interacción entre los factores altitud y temporada $(P<$ 0,$001 ; G . L .=12$ ).

Para C. mexicana se registró mayor producción de hojas a 520 y 1600 msnm que en altitudes de 350 y $90 \mathrm{msnm}$, y el número de hojas más bajo se obtuvo a 1960 msnm (Tabla 5, Figura 10 a-d). En cambio para C. boissieri (Tabla 5, Figura 11 a-d) y E. ebano (Tabla 5, Figura 12 ad) se encontró mayor número de hojas en las cuatro primeras elevaciones y menor en la localidad de mayor altitud. La especie introducida $M$. azederach (Tabla 5, Figura 13 a-d) mostró mayor número de hojas en las altitudes normales de su distribución en la región (a 520, 350 y 90 msnm), que en las dos elevaciones mayores.

En general, se encontró mayor producción de hojas en las altitudes normales de distribución de las especies evaluadas (a 90, 350 y $520 \mathrm{msnm}$ ), debido probablemente a la ocurrencia de temperaturas más altas que las que se presentaron en las altitudes mayores. Estos resultados coinciden con Klanderud (2005), quien reportó una interacción positiva entre los efectos de mayores 
Tabla 4. Promedios de longitud de plantas para cuatro especies en localidades de diferente altitud.

\begin{tabular}{|c|c|c|c|c|c|c|c|c|}
\hline \multirow{3}{*}{$\begin{array}{l}\text { Altitud } \\
\text { (m) }\end{array}$} & \multicolumn{8}{|c|}{ Promedios de longitud de plantas $(\mathrm{mm})$} \\
\hline & \multicolumn{2}{|c|}{ C. mexicana } & \multicolumn{2}{|c|}{ C. boissieri } & \multicolumn{2}{|l|}{ E. ebano } & \multicolumn{2}{|c|}{ M. azederach } \\
\hline & $\begin{array}{l}\text { Mayor } \\
\text { promedio }\end{array}$ & $\begin{array}{l}\text { Menor } \\
\text { promedio }\end{array}$ & $\begin{array}{l}\text { Mayor } \\
\text { promedio }\end{array}$ & $\begin{array}{l}\text { Menor } \\
\text { promedio }\end{array}$ & $\begin{array}{l}\text { Mayor } \\
\text { promedio }\end{array}$ & $\begin{array}{l}\text { Menor } \\
\text { promedio }\end{array}$ & $\begin{array}{l}\text { Mayor } \\
\text { promedio }\end{array}$ & $\begin{array}{l}\text { Menor } \\
\text { promedio }\end{array}$ \\
\hline 90 & 216,5 a & $140,8 \mathrm{de}$ & $91,0 \mathrm{abc}$ & $75,0 \mathrm{bcd}$ & 195,0 a & $177,0 \mathrm{ab}$ & 225,2 a & $183,5 \mathrm{~cd}$ \\
\hline 350 & 219,0 a & $137,0 \mathrm{de}$ & 94,6 a & $82,8 \mathrm{abc}$ & 195,0 a & $179,0 a b$ & 219,7 a & $184,9 \mathrm{~cd}$ \\
\hline 520 & 237,3 a & $165,0 \mathrm{bcd}$ & $95.0 \mathrm{a}$ & $84,0 \mathrm{abc}$ & 198,0 a & $184,0 \mathrm{ab}$ & 228,0 a & $190,1 \mathrm{bcd}$ \\
\hline 1600 & 233,5 a & $83,16 \mathrm{fg}$ & $91,0 \mathrm{abc}$ & 54,0 cde & 199,0 a & $79,0 \mathrm{c}$ & 192,6 bc & $55,0 \mathrm{fg}$ \\
\hline 1960 & 125,0 def & $42,00 \mathrm{~g}$ & 32,0 def & $14,0 \mathrm{f}$ & $142,1 \mathrm{~b}$ & $37,0 \mathrm{~d}$ & 150,0 e & $24,0 \mathrm{~g}$ \\
\hline
\end{tabular}

Letras distintas indican diferencia estadística significativa, de acuerdo a pruebas de comparación de medias de Tukey $(\alpha=0,05)$.

Tabla 5. Número de hojas promedio por planta para cuatro especies en diferentes altitudes.

\begin{tabular}{|c|c|c|c|c|c|c|c|c|}
\hline \multirow{3}{*}{$\begin{array}{l}\text { Altitud } \\
(\mathrm{m})\end{array}$} & \multicolumn{8}{|c|}{ Número de hojas } \\
\hline & \multicolumn{2}{|c|}{ C. mexicana } & \multicolumn{2}{|c|}{ C. boissieri } & \multicolumn{2}{|l|}{ E. ebano } & \multicolumn{2}{|c|}{ M. azederach } \\
\hline & $\begin{array}{l}\text { Mayor } \\
\text { promedio }\end{array}$ & $\begin{array}{l}\text { Menor } \\
\text { promedio }\end{array}$ & $\begin{array}{l}\text { Mayor } \\
\text { promedio }\end{array}$ & $\begin{array}{l}\text { Menor } \\
\text { promedio }\end{array}$ & $\begin{array}{l}\text { Mayor } \\
\text { promedio }\end{array}$ & $\begin{array}{l}\text { Menor } \\
\text { promedio }\end{array}$ & $\begin{array}{l}\text { Mayor } \\
\text { promedio }\end{array}$ & $\begin{array}{l}\text { Menor } \\
\text { promedio }\end{array}$ \\
\hline 90 & 28,9 a & $16,4 \mathrm{bc}$ & 9,91 a & $8,75 a b$ & 18,97 a & 14,95 a & $25,5 a b$ & 17,5 abcde \\
\hline 350 & 29,3 a & $16,5 \mathrm{bc}$ & 9,50 a & $8,67 a b$ & 19,78 a & 17,46 a & $27,0 a b$ & $19,3 \mathrm{abcd}$ \\
\hline 520 & $30,7 \mathrm{a}$ & $20,3 a b c$ & $10,0 \mathrm{a}$ & $8,50 a b$ & 19,65 a & 16,97 a & $28,0 a$ & 18,8 abcd \\
\hline 1600 & $30,1 \mathrm{a}$ & $12,1 \mathrm{~cd}$ & 9,19 a & $4,00 \mathrm{~cd}$ & 15,47 a & $7,5 \mathrm{~b}$ & $19,1 \mathrm{abcd}$ & 7,50 efg \\
\hline 1960 & 14,2 bc & $3,2 \mathrm{~d}$ & $5,33 \mathrm{bc}$ & $0,78 \mathrm{~d}$ & 14,10 a & $2,00 \mathrm{~b}$ & 16,0 bcdef & $3,90 \mathrm{~g}$ \\
\hline
\end{tabular}

Letras distintas indican diferencia estadística significativa, de acuerdo a pruebas de comparación de medias de Tukey $(\alpha=0,05)$.

temperaturas y la producción de hojas, en ecosistemas alpinos. Además, también se ha reportado mayor crecimiento en área foliar y biomasa como efectos asociados a más altas temperaturas (Morison y Lawlor, 1999; Davis et al., 2002).

\section{Comparación entre especies y entre elevaciones}

Debido principalmente al suministro de humedad y a las altas temperaturas,
C. mexicana, C. boissieri, E. ebano y $M$. azederach tuvieron mayor emergencia de plántulas dentro de su área de distribución (a 90, 350 y 520 msnm) y por encima de ésta (a $1600 \mathrm{msnm}$ ). El crecimiento de plántulas y el número de hojas también fue mayor dentro de sus límites naturales de distribución. El sitio a 1600 msnm mostró altos valores de emergencia de plántulas, crecimiento y producción de hojas en las temporadas de primavera 2003 y primavera 2004, por lo que se considera que este sitio podría servir para 

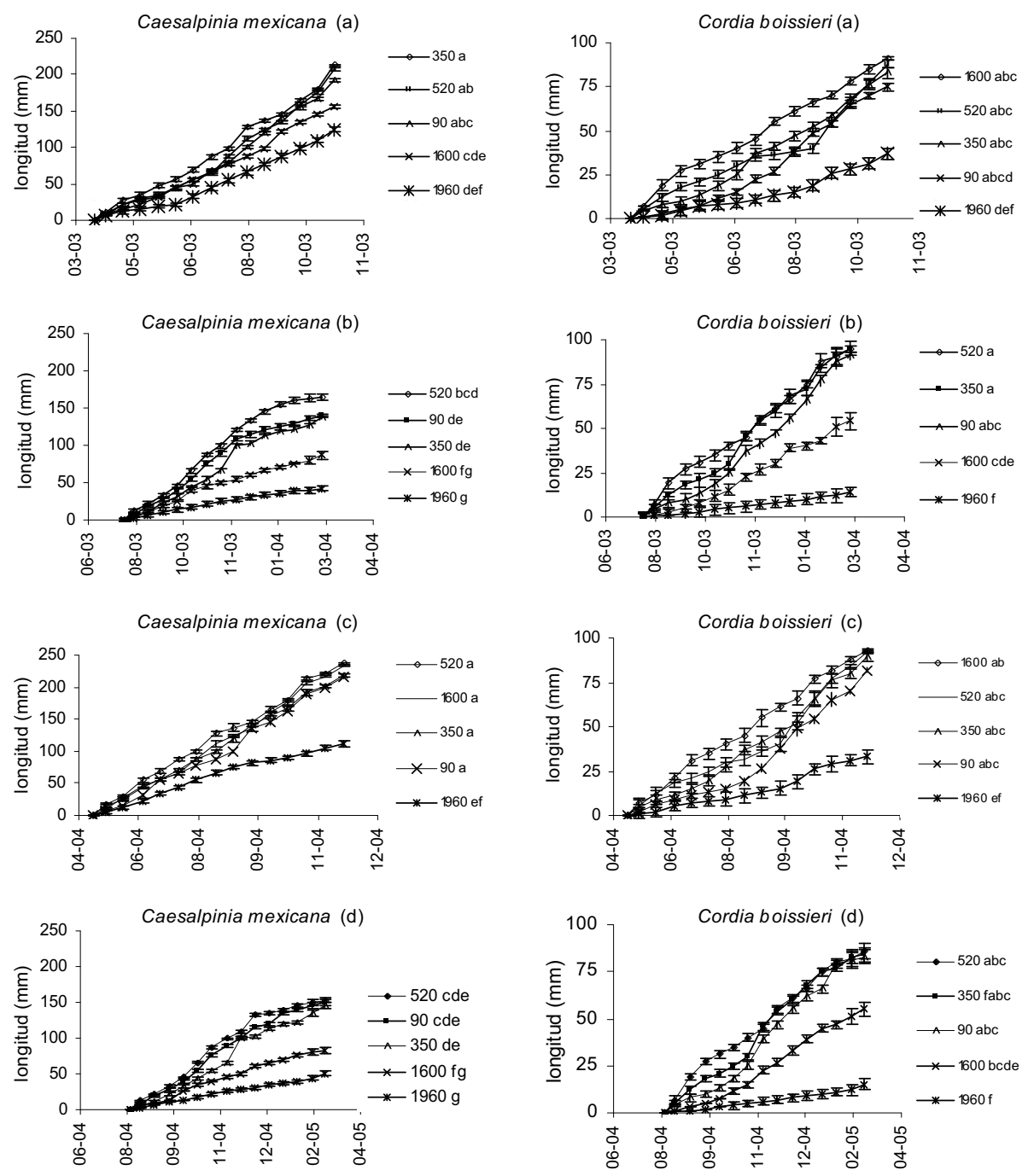

Figura 6.

Promedios de longitud de plantas $(\mathrm{mm})$ para C. mexicana por cuatro temporadas de crecimiento (a-d) en cinco altitudes de 90 a 1960 msnm. Cada punto representa los promedios de porcentaje de germinación acumulada, en seis repeticiones de 20 semillas por cada repetición. Letras diferentes indican diferencia estadística significativa, de acuerdo a pruebas de comparación de medias de Tukey $(\alpha=0,05)$ realizada a los 7 meses. Las barras de error (+/-) representan intervalos de confianza para cada media $(\alpha=0,05)$.

Figura 7.

Promedios de longitud de plantas $(\mathrm{mm})$ para $C$. boissieri por cuatro temporadas de crecimiento (a-d) en cinco altitudes de 90 a 1960 msnm. Cada punto representa los promedios de porcentaje de germinación acumulada, en seis repeticiones de 20 semillas por cada repetición. Letras diferentes indican diferencia estadística significativa, de acuerdo a pruebas de comparación de medias de Tukey $(\alpha=0,05)$ realizada a los 7 meses. Las barras de error (+/-) representan intervalos de confianza para cada media $(\alpha=0,05)$. 

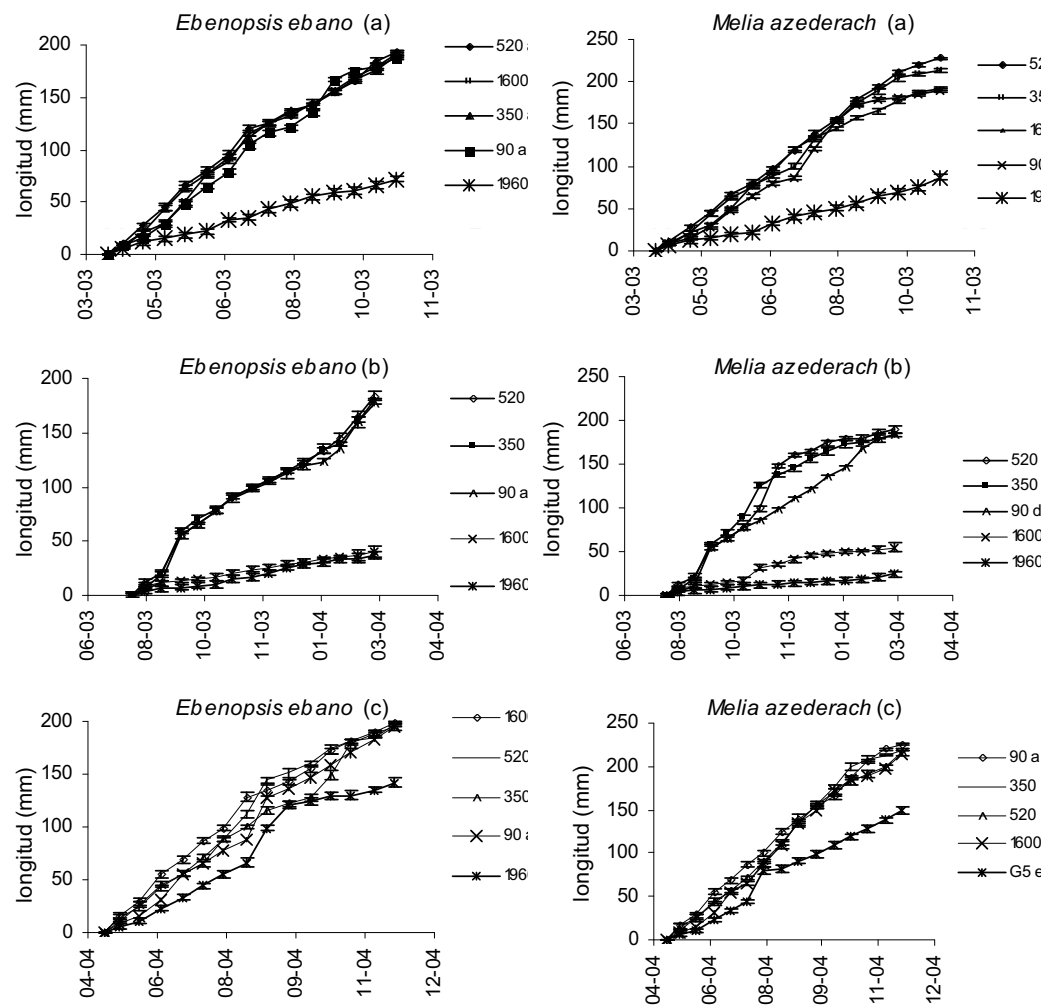

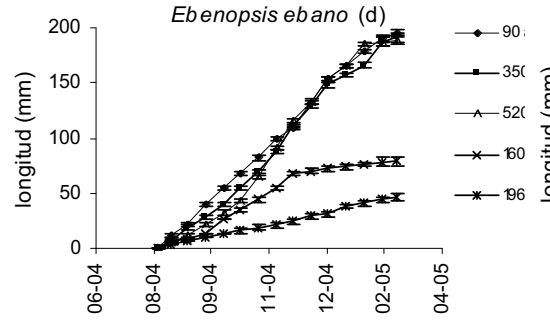

Figura 8.

Promedios de longitud de plantas ( $\mathrm{mm}$ ) para E. ebano por cuatro temporadas de crecimiento (a-d) en cinco altitudes de 90

a $1960 \mathrm{msnm}$. Cada punto representa los promedios de porcentaje de germinación acumulada, en seis repeticiones de 20 semillas por cada repetición. Letras diferentes indican diferencia estadística significativa, de acuerdo a pruebas de comparación de medias de Tukey $(\alpha=0,05)$ realizada a los 7 meses. Las barras de error (+/-) representan intervalos de confianza para cada media $(\alpha=0,05)$.

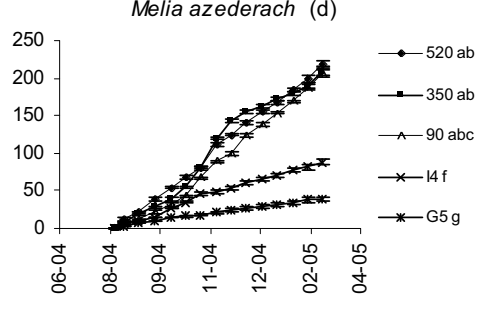

Figura 9.

Promedios de longitud de plantas $(\mathrm{mm})$ para $M$. azederach por cuatro temporadas de crecimiento (a-d) en cinco altitudes de 90 a 1960 msnm. Cada punto representa los promedios de porcentaje de germinación acumulada, en seis repeticiones de 20 semillas por cada repetición. Letras diferentes indican diferencia estadística significativa, de acuerdo a pruebas de comparación de medias de Tukey $(\alpha=0,05)$ realizada a los 7 meses. Las barras de error (+/-) representan intervalos de confianza para cada media $(\alpha=0,05)$. 

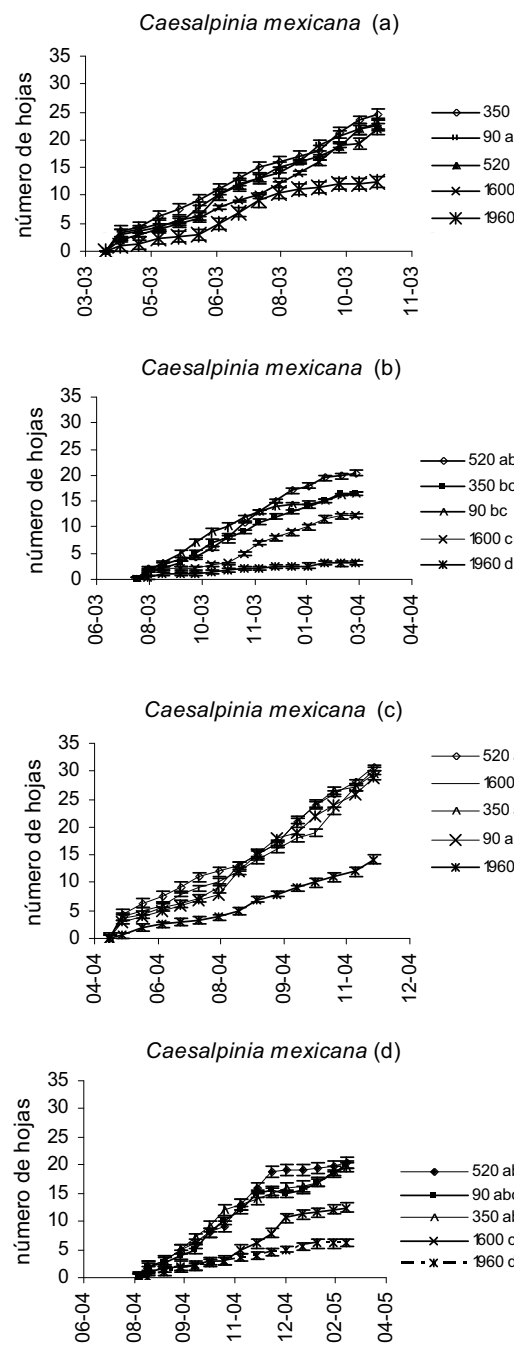

Figura 10.

Promedios de producción de hojas para C. mexicana por cuatro temporadas de crecimiento (a-d) en cinco altitudes de 90

a $1960 \mathrm{msnm}$. Cada punto representa

los promedios de porcentaje de germinación acumulada, en seis repeticiones de 20 semillas por cada repetición. Letras diferentes indican diferencia estadística significativa, de acuerdo a pruebas de comparación de medias de Tukey $(\alpha=0,05)$ realizada a los 7 meses. Las barras de error (+/-) representan intervalos de confianza para cada media $(\alpha=0,05)$.
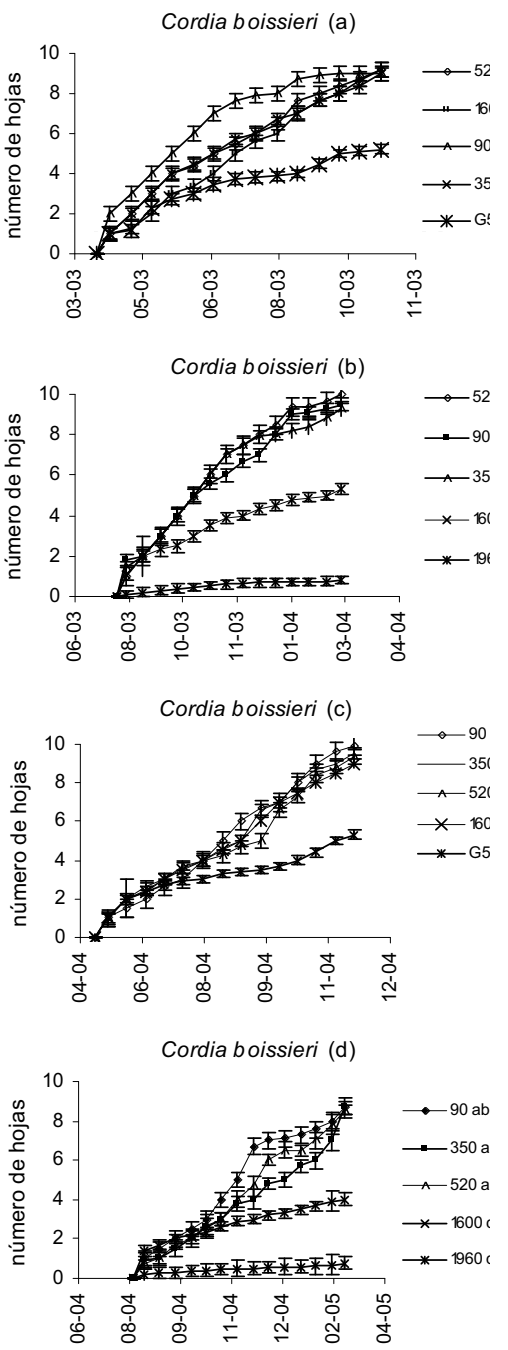

Figura 11

Promedios de producción de hojas para C. boissieri por cuatro temporadas de crecimiento (a-d) en cinco altitudes de 90

a $1960 \mathrm{msnm}$. Cada punto representa los promedios de porcentaje de germinación acumulada, en seis repeticiones de 20 semillas por cada repetición. Letras diferentes indican diferencia estadística significativa, de acuerdo a pruebas de comparación de medias de Tukey $(\alpha=0,05)$ realizada a los 7 meses. Las barras de error (+/-) representan intervalos de confianza para cada media $(\alpha=0,05)$. 



Figura 12.

Promedios de producción de hojas para,

E. ebano por cuatro temporadas (a-d) en cinco altitudes de 90 a 1960 msnm.

Cada punto representa los promedios de porcentaje de germinación acumulada, en seis repeticiones de 20 semillas por cada repetición. Letras diferentes indican diferencia estadística significativa, de acuerdo a pruebas de comparación de medias de Tukey $(\alpha=0,05)$ realizada a los 7 meses. Las barras de error (+/-) representan intervalos de confianza para cada media $(\alpha=0,05)$.

Figura 13

Promedios de producción de hojas para $M$. azederach por cuatro temporadas (a-

d) en cinco altitudes de 90 a 1960 msnm. Cada punto representa los promedios de porcentaje de germinación acumulada, en seis repeticiones de 20 semillas por cada repetición. Letras diferentes indican diferencia estadística significativa, de acuerdo a pruebas de comparación de medias de Tukey

$(\alpha=0,05)$ realizada a los 7 meses. Las barras de error (+/-) representan intervalos de confianza para cada media $(\alpha=0,05)$. 
el desplazamiento altitudinal de las especies, pero únicamente en primavera, aunque las respuestas de las especies al calentamiento global pueden ser contrastantes. Por ejemplo, Jensen (2003) reportó una reducción y ampliación de las áreas de distribución de diversas especies; asimismo, Rehfeldt et al. (2002), encontraron cambios en la presencia y abundancia de Pinus sylvestris por causa del cambio climático. En otros estudios, se reportaron riesgos de extinción por la reducción en la capacidad de las plantas para resistir y recuperarse de perturbaciones ambientales, como plagas y enfermedades Jump y Peñuelas, 2005; McLaughlin et al., 2002).

El sitio a 1960 msnm presentó los valores más bajos para todas las especies en todos los parámetros objeto de estudio, debido probablemente a las bajas temperaturas. Sin embargo, existen otros factores que pueden estar limitando la colonización latitudinal y altitudinal de las especies, como la fragmentación y pérdida de hábitats (Dirnböck et al., 2003), o bien, el establecimiento de plantaciones comerciales (Alig et al., 2002; Hewitt y Kellman, 2002).

\section{CONCLUSIONES}

En las tres especies nativas y en la especie introducida, los mayores porcentajes de emergencia de plántulas se presentaron en su área de distribución natural (altitudes de 90, 350 y 520 msnm), y por encima de ésta (a $1600 \mathrm{msnm}$ ), principalmente durante las primaveras de 2003 y 2004. Los menores porcentajes se registraron a 1960 msnm. Además, el crecimiento de plántulas y la producción de hojas también fueron mayores dentro del área de distribución natural.

La temporada en que se registraron los valores más altos de los parámetros estudiados fue en la primavera de 2004. De las cuatro especies evaluadas, $C$. mexicana mostró mayor porcentaje de emergencia, mayor crecimiento y mayor cantidad de hojas, seguida de E. ebano, M. azederach y C. boissieri.

Las elevaciones de 90,350 y 520 msnm presentan condiciones aptas para la producción de plantas, las cuales podrian utilizarse en programas de reforestación y restauración de ecosistemas forestales. A $1600 \mathrm{msnm}$ es recomendable el inicio de producción de plantas en primavera.

Los resultados de la presente investigación sugieren que las especies estudiadas tienen la capacidad para germinar y crecer por encima de su área de distribución actual.

\section{RECONOCIMIENTOS}

La presente investigación fue apoyada por CONACYT 44806-Q y 112284; PAICYT CN529-01, CN-515-01, CN904-04, CN1134-05.

\section{REFERENCIAS}

Alanís. G. 1981. Aprovechamiento de la flora nativa en el estado de Nuevo León. In: González Vicente, C.E., I. Casas Días y R. Padilla Ibarra (Editores). Memoria de la Primera Reunión sobre Ecología, Manejo y Domesticación de Plantas Útiles del Desierto. Instituto Nacional de Investigaciones Forestales, SARH, Monterrey. Publicación Especial 31:220-227.

Alig, R. J., D. M. Adams y B. A. McCarl. 2002. Projecting impacts of global climate change on the US forest and agricultural sectors and carbon 
budgets. For. Ecol. Manage. 169:3-14.

Bret-Harte, M. S., E. A. García, V. M. Sacré, J. R. Whorley, J. L. Wagner, S. C. Lippert y S. Chapin III. 2004. Plant and soil responses to neighbour removal and fertilization in Alaskan tussock tundra. J. Ecol. 92:635-647.

Castro, J., R. Zamora, J.A. Hódar y J.M. Gómez. 2004. Seedling establishment of a boreal tree species (Pinus sylvestris) at its southernmost distribution limit: consequences of being in a marginal mediterranean habitat. J. Ecol. 92: 266-277.

Clark, J. S. 1998. Why trees migrate so fast: confronting theory with dispersal biology and the paleorecord. Am. Nat. 152:204-224.

Davis, M., S. Pritchard, R. Mitchell, S. Prior, H. Rogers y B. Runion. 2002. Elevated atmospheric $\mathrm{CO}_{2}$ affects structure of a model regenerating longleaf pine community. J. Ecol. 90:130-140.

Dirnböck, T., S. Dullinger y G. Grabherr. 2003. A regional impact assessment of climate and land-use change on alpine vegetation. J. Biogeogr. 30:401-417.

Escudero, A., Carnes, L. F. y PérezGarcía, F. 1997. Seed germination of gypsophytes and gypsovags in semiarid central Spain. Journal of Arid Environment 36: 487-497.

Estrada, E. y J. Marroquín. 1991. Leguminosas en el centro-sur de Nuevo León. Facultad de Ciencias Forestales. UANL. Rep. Cient. No. 10 (especial), Linares N.L. México, 258 pp.
Fenner, M. y K. Thompson. 2005. The ecology of seeds. Cambridge University Press, Cambridge. 250 pp.

Flores J. y O. Briones. 2001. Plant lifeform and germination in a mexican inter-tropical desert: effects of soil water potential and temperature. J. Arid Environ. 47:485-497.

Flores, J. y E. Jurado. 1998. Germination and early growth traits of 14 plant species native to northern Mexico. Southw. Nat. 43:40-46.

Foroughbakhch, P. R. 1989. Tratamiento a la semilla de catorce especies de uso múltiple de zonas de matorral y su influencia en la germinación. Rep. Cient. No. 11, Facultad de Ciencias Forestales, UANL, Linares N.L. México. 20 pp.

García, J. y E. Jurado. 2003. Influence of plant cover on germination in matorral in northeastern Mexico. For. Ecol. Manag. 177:11-16.

Grabherr, G., M. Gottfried y H. Pauli. 1994. Climate effects on mountain plants. Nature 369:448.

Heiseke, D. y R. Foroughbakhch. 1985. El matorral como recurso forestal. Reporte Científico No. 1. Facultad de Silvicultura y Manejo de Recursos Naturales, U.A.N.L., Linares N.L. México, $31 \mathrm{pp}$.

Hewitt, N. y M. Kellman. 2002. Tree seed dispersal among forest fragments: I. Conifer plantations as seed traps. J. Biogeogr. 29:337-349.

Higgins, S.I., J.S. Clark, R. Nathan, T. Hovestadt, F. Schurr, J.M.V. Fragoso, M.R. Aguiar, E. Ribbens y S. Lavorel. 2003. Forecasting plant migration rates: managing uncer- 
tainty for risk assessment. J. Ecol. 91:41-347.

Hilhorst, H. W. M. y C. M. Karssen. 2000. Effect of chemical environment on seed germination. En: M. Fenner (Editor), Seeds: the ecology of regeneration in plant communities, 2nd edn, pp.293-310. CABI Publishing, Wallingford, UK.

Holtmeier, F. K. y G. Broll. 2005. Sensitivity and response of northern hemisphere altitudinal and polar treelines to environmental change at landscape and local scales. Glob. Ecol. Biogeogr. 14:395-410.

Hughes, L. 2000. Biological consequences of global warming: is the signal already apparent? Trends Ecol. Evol. 15:56-61.

Jensen, M. W. 2003. Consensus on ecological impacts remains elusive. Science 299:38.

Jurado E, J.F. García, J. Flores y E. Estrada. 2006. Leguminous seedling establishment in tamaulipan thornscrub of northeastern Mexico. For. Ecol. Manage. 221:133-139.

Jurado, E., J. Flores, J. Navar y J. Jiménez. 1998. Seedling establishment under native tamaulipan thornscrub and Leucaena leucocephala plantation. For. Ecol. Manage. 105:151-157.

Jurado, E., O. Aguirre, J. Flores, J. Navar, J. Jiménez, H. Villalón y D. Wester. 2000. Germination in tamaulipan thornscrub of northeastern Mexico. J. Arid Environ. 46:413-424.

Jump A.S. y J. Peñuelas. 2005. Running to stand still: adaptation and the response of plants to rapid climate change. Ecol. Lett. 8:1010-1020.
Klanderud, K. 2005. Climate change effects on species interactions in an alpine plant community. J. Ecol. 93:127-137.

Kullman, L. 2002. Rapid recent rangemargin rise of tree and shrub species in the swedish scandes. J. Ecol. 90:68-77.

McLaughlin, J. F., J. J. Hellman, C. L. Boggs y P. R. Ehrlich. 2002. Climate change hastens population extinctions. Proc. Natl. Acad. Sci. U.S.A. 99:6070-6074.

Morison, J. I. L. y D. W. Lawlor. 1999. Interactions between increasing $\mathrm{CO}_{2}$ concentration and temperature on plant growth. Plant, Cell Environ. 22:659-682.

Niembro R., A. 1986. Árboles y arbustos útiles de México. Limusa. 206 p.

Parmesan, C. 1996. Climate and species' range. Nature 382:765-766

Parmesan, C. y G. Yohe. 2003. A globally coherent fingerprint of climate change impacts across natural systems. Nature 421:37-42.

Pérez-García, F. y González-Benito, M. E. 2005. Seed germination of five Helianthemum species: Effect of temperature and presowing treatments. Journal of Arid Environment 65 (4): 688-693.

Pérez-García, F., Iriondo, J. M., González-Benito, M. E., Carnes, L. F., Tapia, J., Prieto, C., Plaza, R. y Pérez, C. 1995. Germination studies in endemic plant species of the Iberian Peninsula. Israel Journal of Plant Science 43: 239-247. 
Polunin, O. y A. Stainton. 1984. Flowers of the Himalayas. Oxford University Press, Oxford. 547 p.

Rehfeldt, G. E., N. M. Tchebakava, Y. I. Parfenova, W. R. Wykoff, N. A. Kuzmina y L. I. Milyutin. 2002. Intraspecific responses to climate in Pinus sylvestris. Glob. Change Biol. 8:912-929.

Reid, N., J. Marroquín y P. Beyer-Münzel. 1990. Utilization of shrubs and trees for browse, fuelwood and timber in the tamaulipan thornscrub, northeastern Mexico. For. Ecol. Manage. 36:61-79.

Sokal, R. R. y F. J. Rohlf. 1994. Biometry; the principles and practice of statistics in biological research. W. $\mathrm{H}$. Freeman, New York. 887 pp.

Tape, K., M. Sturm y C. Racine. 2006. The evidence for shrub expansion in northern Alaska and the Pan-Arctic. Glob. Change Biol. 12:686-702.

Téllez, R. y R. Foroughbakhch. 1990. Plantas aprovechadas por el ganado caprino en una zona de matorral mediano espinoso del noreste de México. Reporte Científico No. 21, Facultad de Ciencias Forestales, Universidad Autónoma de Nuevo León. 37 pp.
Thomas, C. D., E. J. Bodsworth, R. J. Wilson, A. D. Simmons, Z. G. Davies, M. Musche y L. Conradt. 2001. Ecological and evolutionary processes at expanding range margins. Nature 411:577-581.

Thompson, K. y J. P. Grime. 1983. A comparative study of germination responses to diurnally-fluctuating temperatures. J. Appl. Ecol. 20:141156.

Udvary, M. D. F. 1975. A classification of the biogeographical provinces of the world.IUCN, Morges, 446 Switzerland, Occas. Paper No. 18, 48 pp.

Vines, R. A. 1984. Trees, shrubs and woody vines of the southwest. University of Texas Press, Austin. pp. 881-882.

Walther, G. R., E. Post, P. Convey, A. Menzel, C. Parmesan, T. J. C. Beebee, J. M. Fromentin, O. HoeghGuldberg y F. Bairlein. 2002. Ecological responses to recent climate change. Nature 416:389-395.

Zamarripa R. 2002. Propiedades físicas y físico mecánicas del canelón Melia azederach L. bajo dos condiciones de crecimiento. Tesis, FCF-UANL. pp. 11-12.

Manuscrito recibido el 13 de septiembre de 2005

Aceptado el 15 de mayo de 2006

Este documento debe citarse como:

García Pérez, J. F., O. Aguirre Calderón, E. Estrada Castillón, J. Flores Rivas, J. Jiménez Pérez y E. Jurado Ybarra. 2007.Germinación y establecimiento de plantas nativas del matorral tamaulipeco y una especie introducida en un gradiente de elevación. Madera y Bosques 13(1):99-117. 